\title{
PhiMiSci
}

Philosophy and the Mind Sciences

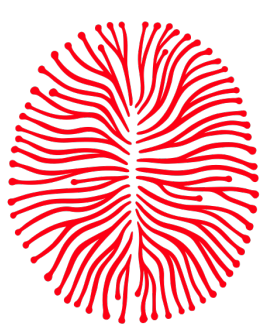

\section{Brain structural complexity and consciousness}

\author{
Chen Song ${ }^{\mathrm{a}}$ (D) (SongC5@cardiff.ac.uk)
}

\begin{abstract}
Structure shapes function. Understanding what is structurally special about the brain that allows it to generate consciousness remains a fundamental scientific challenge. Recently, advances in brain imaging techniques have made it possible to measure the structure of human brain, from the morphology of neurons and neuronal connections to the gross anatomy of brain regions, in-vivo and non-invasively. Using advanced brain imaging techniques, it was discovered that the structural diversity between neurons and the topology of neuronal connections, as opposed to the sheer number of neurons or neuronal connections, are key to consciousness. When the structural diversity is high and the connections follow a modular topology, neurons will become functionally differentiable and functionally integrable with one another. The high levels of differentiation and integration, in turn, enable the brain to produce the richest conscious experiences from the smallest number of neurons and neuronal connections. Consequently, across individuals, those with a smaller brain volume but a higher structural diversity tend to have richer conscious experiences than those with a larger brain volume but a lower structural diversity. Moreover, within individuals, a reduction in neuronal connections, if accompanied by an increase in structural diversity, will result in richer conscious experiences, and vice versa. These findings suggest that having a larger number of neurons and neuronal connections is not necessarily beneficial for consciousness; in contrast, an optimal brain architecture for consciousness is one where the richest conscious experiences are generated from the smallest number of neurons and neuronal connections, at the minimal cost of biological material, physical space, and metabolic energy.
\end{abstract}

\section{Keywords}

Brain structural complexity $\cdot$ Consciousness $\cdot$ Individual differences $\cdot$ Multimodal brain imaging Sleep

This article is part of a special issue on "The Neural Correlates of Consciousness," edited by Sascha Benjamin Fink and Ying-Tung Lin.

\footnotetext{
${ }^{a}$ Cardiff University Brain Research Imaging Centre, School of Psychology, Cardiff University, Cardiff, UK 


\section{Introduction}

A central idea in biology is that structure determines function, as Jean Fernel (Tubbs, 2015), the founder of modern anatomy and physiology, famously said, "anatomy (the study of body structure) is to physiology (the study of body function) as geography is to history; it describes the theatre of events." In emphasizing the close relationship between structure and function, Jean Fernel followed the footstep of Herophilus (Pearce, 2013), the ancient Greek who performed the first dissection of human body and recognized the importance of body structure in determining body function. However, for the nearly two-thousand years between the death of Herophilus and the birth of Jean Fernel, the idea that structure determines function was very much overlooked in the realm of biomedical science. Even today, the structure and function of biological systems are often separately studied, and their relationship much less addressed.

In consciousness research, the structure-function relationship has also been long neglected. Since the introduction of brain imaging techniques a few decades ago, researches have been focused on the functional rather than the structural basis of consciousness, with studies searching for brain regions whose activities correlate with the level or the contents of consciousness (Crick \& Koch, 2003; Koch et al., 2016). This approach overlooks the important fact that the brain is an interconnected entity where the activity of one region would influence the activities of other regions via direct or indirect connections. As such, any correlation between consciousness and the activity of a particular region is possibly mediated by the activities of other regions. Indeed, using this approach, different studies tend to identify different brain regions as the functional correlates of consciousness and the discrepancy has led to the debates among various theories of consciousness (Boly et al., 2017; Fink, 2016; Lau \& Rosenthal, 2011; Odegaard et al., 2017; Tononi et al., 2016).

While the interconnectedness of the brain has posed a great challenge for identifying the functional correlates of consciousness, recent advances in brain imaging techniques (Edwards et al., 2018; Glasser, Smith, et al., 2016; Jones et al., 2018; Panda et al., 2017) have instead made it possible to measure the biophysical structure of human brain non-invasively and study the structural basis of consciousness. In this article, we will review the contributions of brain imaging techniques towards uncovering the relationship between brain structure, brain function, and consciousness. We will provide an overview of advanced brain imaging techniques and discuss how these techniques can unveil brain structural complexity (section 2) and its relationship to consciousness (section 3). 


\section{Understanding brain structural complexity}

In describing the relationship between brain structure and brain function, Jean Fernel (Tubbs, 2015), the founder of modern anatomy and physiology, once said, "the brain is the seat of the mind and its parts; the mind being endowed with numerous faculties, man has rightly been provided with a larger accommodation for it than the other creature possesses, and this accommodation is associated with more instruments." Jean Fernel, like many other scientists, intuitively assumes that having a larger brain volume ("larger accommodation"), or a larger number of neurons and neuronal connections ("more instruments"), is key to having better brain functionality. But are the volume of the brain and the number of neurons truly the key? And is more really better?

The common assumption "the more the better" ignores the costs associated with having a larger brain volume or a larger number of neurons and neuronal connections. Every neuron and every neuronal connection would cost biological material to build, physical space to accommodate, and metabolic energy to sustain (Kaas, 2000). If the same brain functions can be achieved using less biological and physical resources (Oizumi et al., 2014), that is likely to reflect a more advantageous and cost-effective brain architecture. In this sense, more is not better; on the contrary, less can be more.

To achieve the maximal functions using the minimal number of neurons and neuronal connections, the key is for neurons to be functionally differentiable from each other (differentiation), and meanwhile, functionally integrable with one another (integration). If neurons are functionally identical to each other, no matter how many neurons there are, the functions they generate as a whole will be equivalent to the functions of a single neuron. If neurons are not functionally integrable with one another, the functions they generate as a whole will be the linear rather than exponential combination of the functions they generate individually.

By nature, differentiation and integration are not compatible (Tononi et al., 1994; Tononi, 1998; Tononi \& Edelman, 1998), as a stronger interaction between neurons will lead to an increase in their integration but a decrease in their differentiation, and vice versa. Nonetheless, a high level of differentiation and a high level of integration can be achieved at the same time, if the structural diversity between neurons is high and the connections between neurons follow a modular topology. In fact, the structural diversity between neurons (cell diversity) and the topology of neuronal connections (cell-cell interaction), as opposed to the sheer number of neurons (cell number) or the sheer volume of brain (organ size), are what underlie the structural complexity of the brain and what distinguish the brain from other organs. Thanks to the recent advances in brain imaging techniques, the structural diversity between neurons and the topology of neuronal connections in the human brain can now be measured in-vivo and non-invasively. In the following subsections, we will discuss how these structural features contribute to the structural complexity and the functionality of the brain, and how these structural features can in turn be assessed using advanced brain imaging techniques. 


\subsection{Contributions of network topology}

The brain can be viewed as a network of interconnected nodes (Figure 1), where each node is a neuron or a brain region (Bullmore \& Sporns, 2009; Sporns et al., 2005; Sporns \& Betzel, 2016). A key feature that distinguishes the brain from other organs is the complexity of cell-cell interaction, shaped largely by the topology of the network. Generally speaking, a more densely connected network would have a higher level of integration but a lower level of differentiation, and vice versa. Take an all-to-all-connected network as an example: in this network, every node is connected to every other node and therefore integrable with them (high integration); however, as a by-product of the dense connections, every node is also fully synchronized with every other node and therefore not differentiated from them (low differentiation). The level of differentiation can be improved by reducing the connections in the network, but that will come at the price of reduced integration. In this sense, there exists a natural trade-off between differentiation and integration.

Despite the natural trade-off between differentiation and integration, a high level of differentiation and a high level of integration can be achieved at the same time, if the network follows a modular topology (Figure 1), with dense connections between nodes in the same module, and sparse connections between nodes from different modules (Bassett \& Gazzaniga, 2011; Bullmore \& Sporns, 2009; Sporns, 2013; Sporns et al., 2005; Sporns \& Betzel, 2016; Tononi et al., 1994; Tononi, 1998; Tononi \& Edelman, 1998). The dense intra-modular connections facilitate the integration between nodes in the same module, while the sparse inter-modular connections facilitate differentiation between nodes from different modules.

The modular topology has many functional benefits. It creates a balance between singularity and redundancy, whereby different nodes in the network can have distinct functions, yet if a node stops functioning, other nodes in the same module can take over (Tononi et al., 1999). It also creates a balance between dynamicity and staticity, whereby a node undergoing a state transition can spread the transition to other nodes in the same module without influencing nodes in other modules (Pan \& Sinha, 2009). Moreover, compared to other network topologies, a modular topology would cost least connections to produce a high level of integration, and least nodes to produce a high level of differentiation, thereby ensuring high cost-efficacy (Clune et al., 2013; Gallos et al., 2012; Song et al., 2005). Given these functional benefits, the presence of modular topology is often taken as an indicator of a high network complexity (Bassett \& Gazzaniga, 2011; Bullmore \& Sporns, 2009; Sporns, 2013; Sporns et al., 2005; Sporns \& Betzel, 2016).

Such a modular topology is observed in the brain, both at the cellular and at the regional levels. At the cellular level, neurons with similar response properties are densely interconnected and clustered into the same cortical column, whereas neurons with different response properties are sparsely interconnected and distributed into different cortical columns (Figure 1), as illustrated by the orientation column, colour column, or ocular dominance column in visual cortices (Gilbert \& Wiesel, 1989; Kaas, 2012; Mountcastle, 1997; Weliky et al., 1995). At a regional

Song, C. (2021). Brain structural complexity and consciousness. Philosophy and the Mind Sciences, 2, 6. https://doi.org/10.33735/phimisci.2021.9185

(c) (1) (c) The author(s). https://philosophymindscience.org ISSN: 2699-0369 
level, functionally similar regions are densely interconnected and spatially clustered, whereas functionally distinct regions are sparsely interconnected and spatially distant (Figure 1), as illustrated by the clustering of visual regions in occipital cortex, auditory regions in temporal cortex, somatosensory regions in central cortex, multisensory regions in parietal cortex, and executive control regions in frontal cortex (Sporns \& Betzel, 2016).

Notably, the modular topology is observed not just in the brain, but also in systems not typically associated with consciousness, such as the metabolic network or the social network (Girvan \& Newman, 2002; Hartwell et al., 1999; Kashtan \& Alon, 2005; Newman, 2006; Ravasz, 2002; Sole \& Valverde, 2006). Such a ubiquitous presence of modular topology indicates that the network topology on its own cannot give rise to consciousness, and the brain should not be simplified to an abstract network. Indeed, by simplifying the brain to an abstract network, the biophysical structure of nodes or connections and the structural diversity between nodes or connections are largely disregarded, whereas these factors may play a central role in shaping the structural complexity and the functionality of the brain. In what follows, we will discuss the contributions of these factors.

\subsection{Contributions of structural diversity}

No two neurons are identical. The cell diversity, driven largely by the structural differences between neurons, is the other key feature that distinguishes the brain from other organs. At the cellular level, the morphology of neuron cell bodies and the morphology of neuronal connections differ substantially from neuron to neuron. The structural differences shape the functional differences between neurons and provide the basis for neuron type classification (Figure 2). Specifically, the morphology of neuron cell bodies shapes the nature of signal computation, where larger neurons, such as pyramidal cells, can receive signals from a larger number of other neurons and perform signal integration, yet smaller neurons, such as granule cells, can only receive signals from a limited number of other neurons and perform signal relay (Bekkers, 2011; Brown et al., 2008; Chklovskii, 2004). The morphology of neuronal connections, on the other hand, shapes the speed of signal transmission, where axonal connections with larger diameter and/or higher myelination can perform faster signal transmissions, and vice versa (Arancibia et al., 2017; Chereau et al., 2017; Firmin et al., 2014; Horowitz et al., 2015).

At the regional level, the distribution of neurons (cytoarchitecture) and the distribution of neuronal connections (myeloarchitecture) differ substantially from region to region (Amunts et al., 2010; Amunts \& Zilles, 2015; Clarke \& Miklossy, 1990; Palomero \& Zilles, 2019). The structural differences shape the functional differences between brain regions and provide the basis for brain parcellation (Figure 2). Specifically, the regions dominated by granule cells, such as primary visual cortex, are involved in signal relay (Shipp, 2005); the regions dominated by pyramidal cells, such as primary motor cortex, are involved in signal integration (Shipp, 


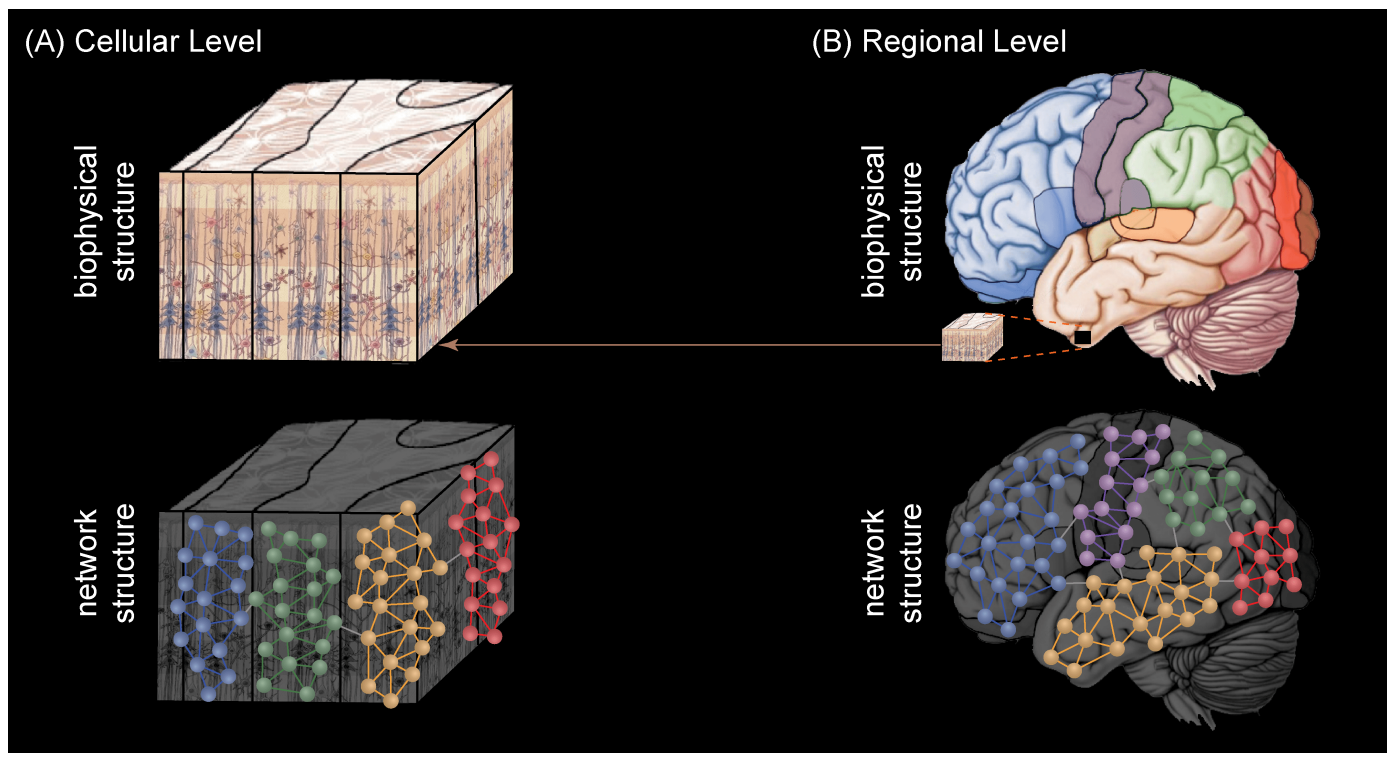

Figure 1: Network Topology in the Brain. The brain can be viewed as a network of interconnected nodes, where each node is a neuron or a brain region. A key feature that distinguishes the brain from other organs is the complexity of cell-cell interaction, shaped largely by the topology of the network. The network at the cellular and the regional levels both follow a modular topology, with dense connections between nodes in the same module (represented by similarly coloured nodes), and sparse connections between nodes from different modules (represented by differently coloured nodes). (A) Specifically, at the cellular level, neurons with similar response properties are densely interconnected and clustered into the same cortical column, whereas neurons with different response properties are sparsely interconnected and distributed into different cortical columns. (B) At the regional level, functionally similar brain regions are often densely interconnected and spatially clustered, whereas functionally distinct brain regions are often sparsely interconnected and spatially distant.

2005); the regions with widespread connections to and from other regions, such as the thalamus and the prefrontal cortex, are involved in signal modulation (Harris et al., 2019; Hwang et al., 2017).

The close relationship between the biophysical structure and the function of the neural systems illustrates why the brain should not be simplified to an abstract network. Under such simplification, the only factor of interest is the topology of the network, whereas the nodes or the connections in the network are treated as abstract units with no intrinsic structure. However, in reality, each node is a neuron or a brain region, and each connection is an axon bundle, all of which has its unique biophysical structure. The biophysical structure of these nodes or connections shapes their functions. Moreover, the structural diversity between these nodes or connections greatly amplifies the structural complexity and enhances the functionality of the brain. 


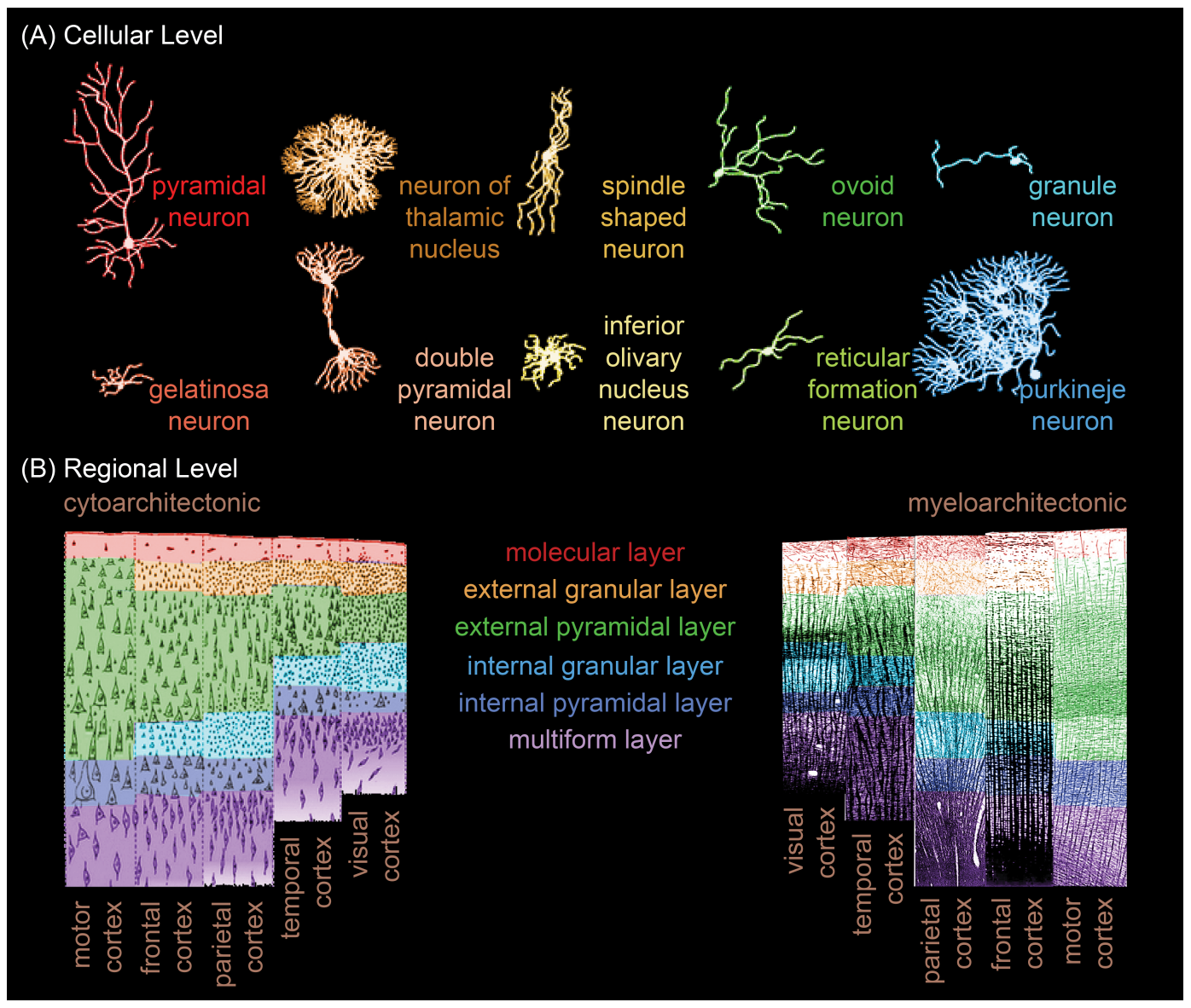

Figure 2: Structural Diversity in the Brain. No two neurons are identical. A key feature that distinguishes the brain from other organs is the cell diversity, driven largely by the structural differences between neurons. (A) At the cellular level, the morphology of neuron cell bodies and the morphology of neuronal connections differ substantially from neuron to neuron. The structural differences shape the functional differences between neurons and provide the basis for neuron type classification. (B) At the regional level, the distribution of neurons (cytoarchitecture) and the distribution of neuronal connections (myeloarchitecture) differ substantially from region to region. The structural differences shape the functional differences between brain regions and provide the basis for the parcellation of brain regions.

The structural diversity can greatly amplify the complexity of the neural network. In particular, a network where the nodes are structurally diverse, compared to a network where the nodes are structurally uniform, will have an exponentially higher complexity level. Take a four-node network as an example: if the nodes had no structural differences and were interchangeable, there would exist six different ways of constructing the network, with one, two, three, four, five, or six connections in the network (Figure 3). However, if the nodes had different structures and were not interchangeable, there would exist sixty-three different ways of constructing the network (Figure 3), including six different ways of constructing a 
one-connection network, fifteen different ways of constructing a two-connection network, twenty different ways of constructing a three-connection network, fifteen different ways of constructing a four-connection network, six different ways of constructing a five-connection network, and one way of constructing a sixconnection network. The linear growth of network complexity with network size in the former case, compared to the exponential growth in the latter case, demonstrates the contribution of structural diversity to the structural complexity of the brain.

The structural diversity also contributes to the joint satisfaction of differentiation and integration. The structural diversity between nodes will lead to the functional diversity between nodes and facilitate their differentiation. The structural diversity between connections, on the other hand, will lead to an uneven signal transmission across the network and facilitate the differentiation among the weakly linked nodes as well as the integration among the strongly linked nodes. Therefore, the structural diversity, on its own and independent of the network topology, can give rise to a high level of differentiation as well as a high level of integration, which in turn enhances the functionality of the brain.

\subsection{Non-invasive imaging of network topology and struc- tural diversity}

Before the introduction of magnetic resonance imaging, the topology of neuronal connections and the structural diversity between neurons were measurable only through histological staining, in-vitro and invasively. With the development of magnetic resonance imaging, magnetic resonance signals can serve as the virtual stains to measure these structural features, in-vivo and non-invasively. In what follows, we will give an overview of how magnetic resonance imaging can be applied to measure the macro-structure and micro-structure of the human brain.

At the macro-structural level, the brain is composed of three major tissues: grey matter, white matter, and cerebrospinal fluid. At the micro-structural level, grey matter is composed of neuron cell bodies, dendrites, and unmyelinated axons (which form the short-distance, intra-regional connections), whereas white matter is composed of myelinated axons (which form the long-distance, inter-regional connections). Cerebrospinal fluid, on the other hand, is the body fluid that provides support for the grey matter and white matter. Due to their differences in cellular composition, grey matter, white matter, and cerebrospinal fluid have different fat and water contents. Utilizing the sensitivity of magnetic resonance signals towards fat and water, the structure of human brain can be non-invasively imaged, with the image intensity values reflecting the gross anatomy of brain regions (macro-structure) or the morphology of neurons and neuronal connections (micro-structure). 


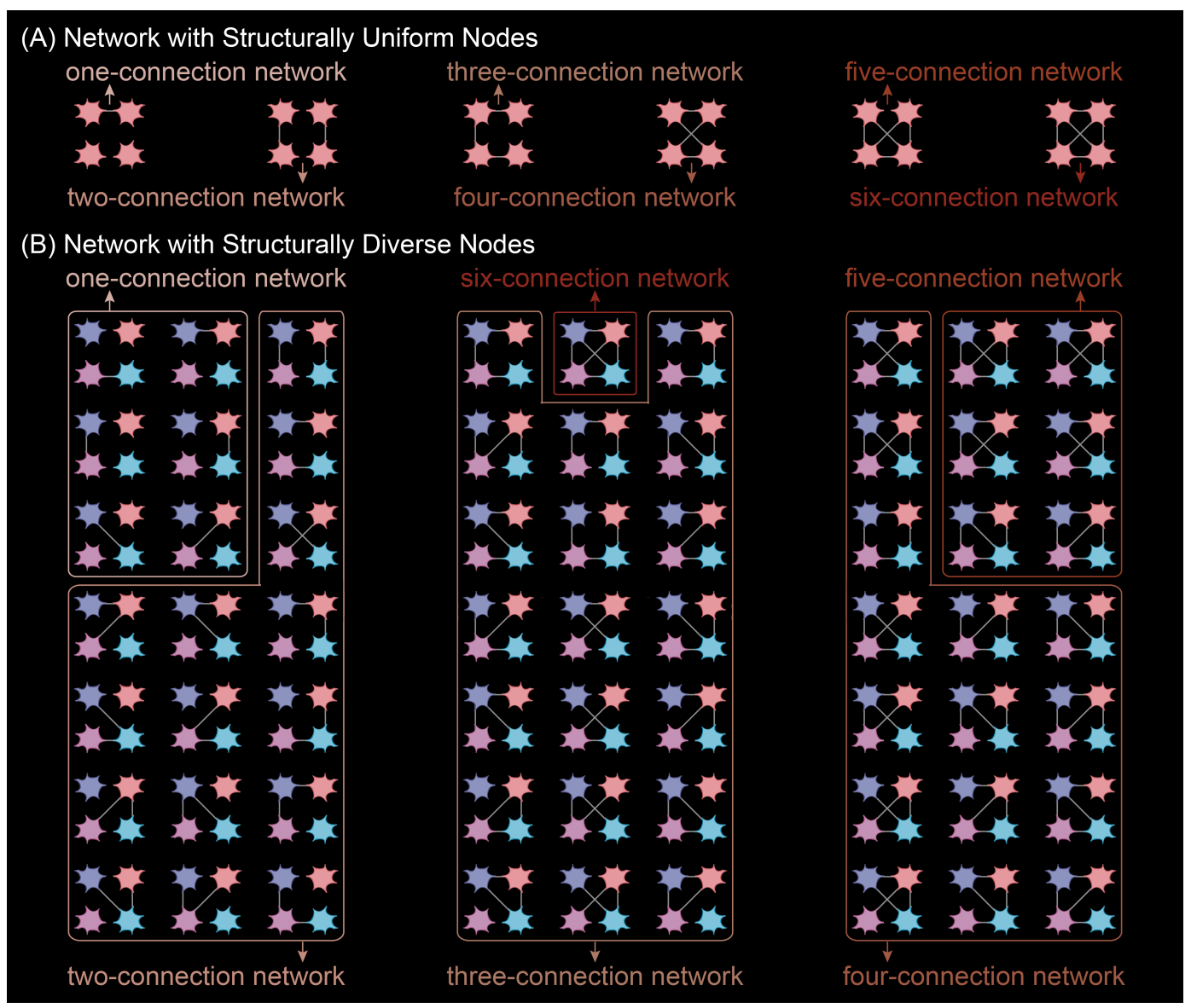

Figure 3: Contributions of Structural Diversity to Network Complexity. A network where the nodes are structurally diverse, compared to a network where the nodes are structurally uniform, will have a complexity level that is exponentially higher. (A) Take a four-node network as an example: if the nodes had no structural differences and were interchangeable, there would exist six different ways of constructing the network, with one, two, three, four, five, or six connections in the network. (B) However, if the nodes had different structures and were not interchangeable, there would exist sixty-three different ways of constructing the network, including six different ways of constructing a one-connection network, fifteen different ways of constructing a two-connection network, twenty different ways of constructing a three-connection network, fifteen different ways of constructing a four-connection network, six different ways of constructing a fiveconnection network, and one way of constructing a six-connection network.

To measure the gross anatomy of brain regions, spin relaxation signal is often acquired, producing brain images where different brain tissues have distinct image intensity values as a result of their differences in fat and water contents (Glasser, Coalson, et al., 2016; Weiskopf et al., 2013). Based on the image intensity values, the brain images can be segmented into grey matter, white matter, and cerebrospinal fluid, from which the three-dimensional brain models can be created (Figure 4). The three-dimensional brain models capture the morphology of the cortex and the 
subcortex, which in turn provide the anatomical landmarks for the parcellation of brain regions (Dale et al., 1999; Fischl et al., 1999). For individual brain region parcellated, its volume and its surface area can be calculated as the summed volume and the summed surface area across all cubic voxels in this region, respectively. For individual cortical location delineated, its thickness can be calculated as the distance between the inner and outer surfaces of the cortex.

To measure the morphology of neurons and neuronal connections, molecular diffusion signal is often acquired, producing brain images that reflect the trajectory of molecular diffusion in brain tissues (Le Bihan \& Iima, 2015). In an unconstrained tissue environment such as the cerebrospinal fluid, the molecular diffusion has an isotropic trajectory. By contrast, in a constrained tissue environment such as the white matter or grey matter, the molecular diffusion in extra-cellular space is partially hindered by the cell membrane, while that in intra-cellular space is fully restricted by the cell membrane (Figure 4). The restricted trajectory of intra-cellular diffusion enables the neuronal morphology, including the size of neuron cell bodies, the diameter of axonal connections, the trajectory of axonal connections, and the branching of dendritic connections, to be measured from molecular diffusion signal (Assaf et al., 2008; Assaf \& Basser, 2005; Palombo et al., 2016, 2018).

\subsection{Brain architecture optimal for functionality}

Taken together, recent advances in brain imaging techniques have made it possible to measure the biophysical structure of human brain, from the morphology of neurons and neuronal connections to the gross anatomy of brain regions, in-vivo and non-invasively. Based on the measures, the volume of brain regions (organ size), the number of neurons (cell number), the structural diversity between neurons (cell diversity), and the topology of neuronal connections (cell-cell interaction) can all be estimated. The structural diversity between neurons (cell diversity) and the topology of neuronal connections (cell-cell interaction), as opposed to the sheer number of neurons (cell number) or the sheer volume of brain (organ size), are what underlie the structural complexity of the brain. When the structural diversity between neurons is high and the connections between neurons follow a modular topology, neurons will become functionally differentiable and functionally integrable with each other at the same time. The high levels of differentiation and integration, in turn, enable the maximal number of functions to be generated from the minimal number of neurons and neuronal connections, at the minimal cost of biological material, physical space, and metabolic energy.

As such, an optimal brain architecture is not necessarily constituted of more neurons or neuronal connections; rather, it is one where the neurons and neuronal connections are structured to produce the highest levels of differentiation and integration. However, is such a brain architecture, optimal for brain functionality, also optimal for consciousness? In the following section, we will look into what constitutes an optimal brain architecture for consciousness. 


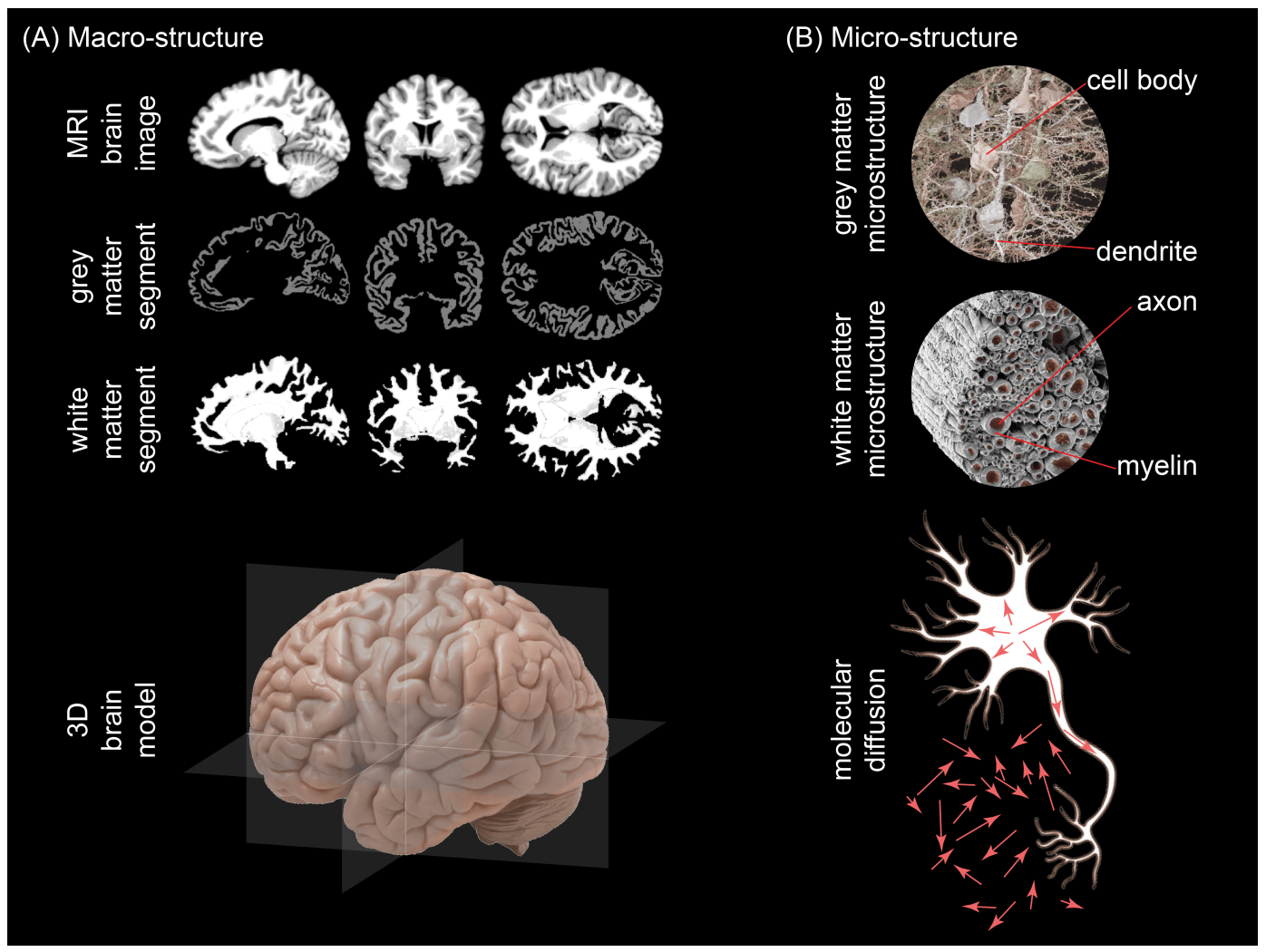

Figure 4: Structural Imaging of the Brain. The brain can be characterized at multiple levels. (A) At the macro-structural level, the brain is composed of three major tissues: grey matter, white matter, and cerebrospinal fluid. To measure the macro-structure of the brain, spin relaxation signal is often acquired, producing brain images where different brain tissues have distinct image intensity values as a result of their differences in fat and water contents. Based on the image intensity values, the brain images can be segmented into grey matter and white matter, from which the three-dimensional brain models can be created. (B) At the micro-structural level, grey matter is composed of neuron cell bodies, dendrites, and unmyelinated axons, whereas white matter is composed of myelinated axons. To measure the micro-structure of the brain, molecular diffusion signal is often acquired, producing brain images that reflect the trajectory of molecular diffusion. The molecular diffusion in extra-cellular space is partially hindered by the cell membrane, while that in intra-cellular space is fully restricted by the cell membrane. The restricted trajectory of intra-cellular diffusion enables the neuronal morphology, including the size of neuron cell bodies, the diameter of axonal connections, the trajectory of axonal connections, and the branching of dendritic connections, to be measured.

\section{From brain structural complexity to conscious- ness}

Consciousness, in theory, is what every one of us should be most familiar with. After all, everything we know about ourselves and about the external world around 
us is via the lens of our own conscious experiences, and the reason why we get to form our unique identity of self and our unique perspective of the world is due to the subjectivity of conscious experiences. However, consciousness, in reality, is probably what the majority of us are most unfamiliar rather than familiar with. We often take consciousness for granted and rarely reflect upon our own conscious experiences; even when we do, different people tend to form different understanding about consciousness, exactly as a result of its subjectivity.

The lack of consensus on consciousness makes it an easy target for philosophic debates, but at the same time, a difficult topic for scientific investigations. The difficulty, first and foremost, is reflected in the conflicting definitions of consciousness. At one end of the spectrum, consciousness has been defined as being aware of and responsive to the external world (Sutherland, 1995). At the other end of the spectrum, consciousness has been equated to being aware of oneself (Lisman, 2017). Both definitions, however, are misleading. Consciousness is literally anything and everything that one experiences, including but not limited to the experience of the external world and the experience of oneself (Koch, 2019). A case in point is dream consciousness, during which one is fully conscious, yet neither of the external world nor necessarily of oneself (Siclari et al., 2013; Windt, 2015).

The inclusiveness of conscious experiences renders it difficult to measure and study. Indeed, if consciousness is any experience and every experience, how can one identify the single brain mechanism that accounts for the variety of conscious experiences? In essence, the mechanism needs to explain visual experience, auditory experience, experience of excitement, experience of sadness, experience of self-esteem, experience of self-doubt, and countless number of other experiences. One possible solution here is to identify the common properties shared by different conscious experiences and search for the brain mechanism that can account for these common properties. In the following subsections, we will discuss what the common properties of conscious experiences are and how, based on these common properties, we can investigate the relationship between brain structural complexity and consciousness.

\subsection{Properties of consciousness}

Consciousness has two properties: differentiation and integration (Tononi et al., 1994; Tononi et al., 2016; Tononi \& Edelman, 1998). No matter what one is experiencing, one's conscious experience is always integrated and structured. The exact way the conscious experience is integrated and structured, as well as the exact contents of conscious experience, however, differ from one conscious experience to the other, which reflects the differentiation aspect of consciousness. As an example, when reading this sentence, the visual appearance of the texts, the semantic meaning of the texts, the thinking triggered by the texts, and the emotions evoked by the text are all parts of an integrated, structured conscious experience. The exact way these parts are integrated and structured, as well as their exact contents, 
are unique to this conscious experience and are what differentiate it from other conscious experiences.

The two properties, differentiation and integration, are also what effectively enrich consciousness (Figure 5). Without the former, consciousness would be reduced to a repertoire of identical, undifferentiable experiences (Figure 5). Without the latter, consciousness would be reduced to a repertoire of unstructured, unintegrated experiences (Figure 5). Therefore, in order for consciousness to be rich, different conscious experiences need to be highly differentiated, and at the same time, individual conscious experience needs to be highly integrated and structured (Tononi et al., 1994; Tononi et al., 2016; Tononi \& Edelman, 1998).

\subsection{Brain architecture optimal for consciousness}

To support the two properties of consciousness, neurons need to be functionally differentiable and functionally integrable with each other at the same time, via which they can produce a set of differentiated yet integrated activity patterns that can then give rise to a repertoire of differentiated yet integrated conscious experiences. All these, in turn, require the structural diversity between neurons to be high and the connections between neurons to follow a modular topology. If neurons are functionally identical to each other, for example as a result of lacking structural diversity or as a result of being over-connected, they will be fully synchronized in their activities and fail to produce differentiated activity patterns; subsequently, consciousness will be reduced to a repertoire of identical, undifferentiable experiences (Figure 5). If neurons are not functionally integrable with one another, for example as a result of being under-connected, they will fail to produce structured and integrated activity patterns; subsequently, consciousness will be reduced to a repertoire of unstructured, unintegrated experiences (Figure 5).

Thus, the structural diversity between neurons and the topology of neuronal connections are not only the very features that distinguish the brain from other organs, underlie brain structural complexity, enhance brain functionality, but also the very factors that give rise to the properties (differentiation, integration) and the richness of consciousness. When the structural diversity between neurons is high and the connections between neurons follow a modular topology, a brain with a smaller volume, less neurons, and less neuronal connections can outperform its counterpart with a larger volume, more neurons, and more neuronal connections, by producing higher structural complexity, better brain functionality, as well as richer conscious experiences.

In this sense, an optimal brain architecture for consciousness is not one with a larger volume, more neurons, or more neuronal connections ("the more the better"); on the contrary, it is one where the largest repertoire of conscious experiences is generated from the smallest number of neurons and neuronal connections, at the minimal cost of biological material, physical space, and metabolic en- 


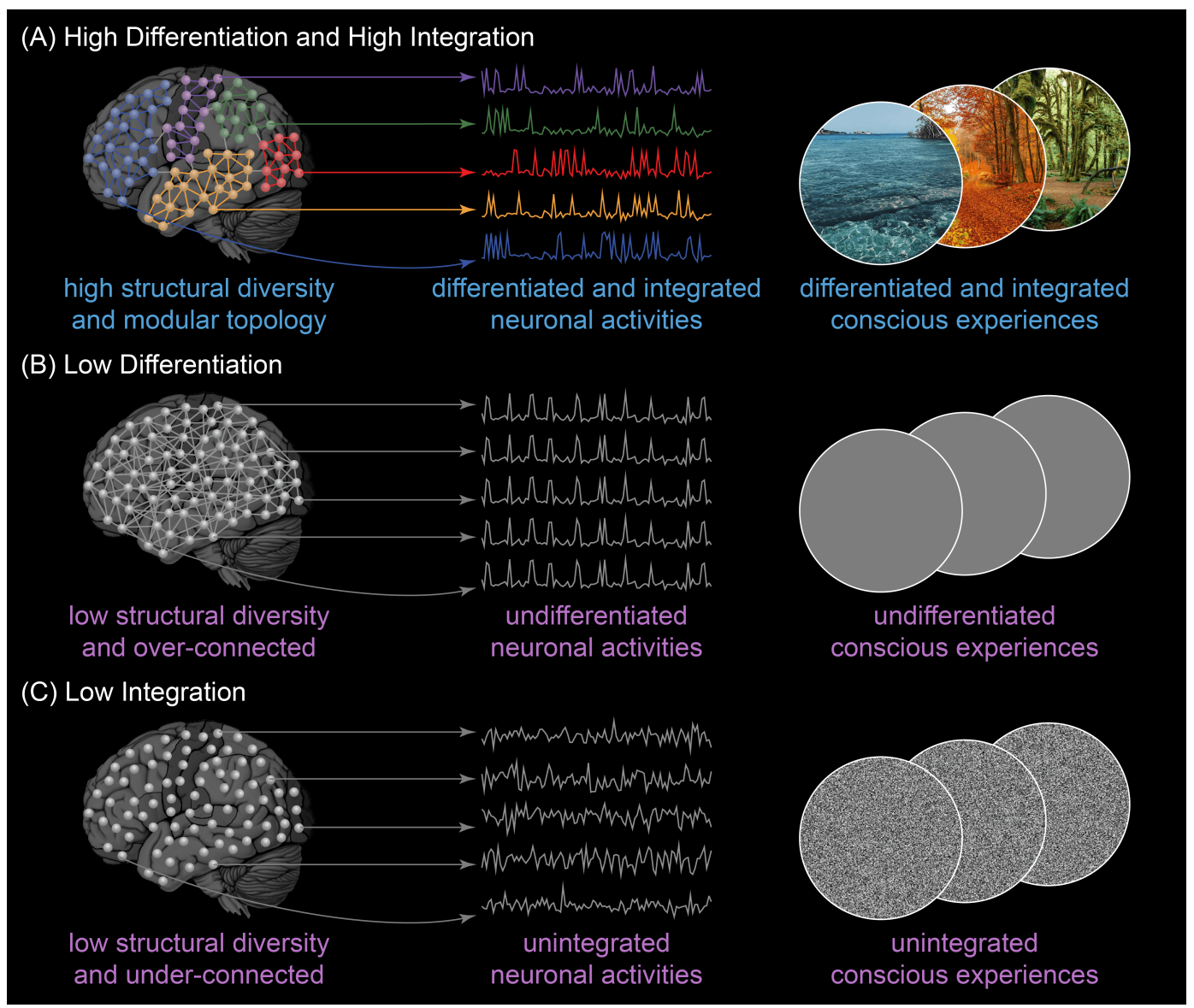

Figure 5: Brain Architecture Optimal for Consciousness. (A) Consciousness has two basic properties: differentiation and integration. To support them, neurons need to be functionally differentiable and functionally integrable with one another, via which they can produce a set of differentiated yet integrated activity patterns that can then give rise to a repertoire of differentiated yet integrated conscious experiences. These, in turn, require the structural diversity between neurons to be high and the connections between neurons to follow a modular topology. (B) If neurons are functionally identical to each other, for example as a result of lacking structural diversity or being over-connected, they will be synchronized in their activities and fail to produce differentiated activity patterns; subsequently, consciousness will be reduced to a repertoire of identical, undifferentiable experiences. (C) If neurons are not functionally integrable with one another, for example as a result of being under-connected, they will fail to produce structured and integrated activity patterns; subsequently, consciousness will be reduced to a repertoire of unstructured, unintegrated experiences.

ergy ("less is more"). The idea "less is more" may appear counter-intuitive; it also contradicts the current practice in the field. Currently, under the common assumption "the more the better," researchers often search for brain region whose volume correlates positively with inter-individual difference or intra-individual change in behavioural performance (Kanai \& Rees, 2011); the observation of positive corre- 
lation is taken as evidence for the involvement of this brain region, whereas the observation of negative correlation is overlooked. By contrast, following the idea "less is more," one would expect the negative correlation between brain volume and behavioural performance to be meaningful.

Despite its counter-intuitiveness, the idea "less is more" has received support from a number of studies, thanks to the advances in brain imaging techniques. These studies reveal that across individuals, those with a smaller brain volume but a higher structural diversity tend to have richer consciousness than those with a larger brain volume but a lower structural diversity; moreover, within individuals, a reduction in neuronal connections, if accompanied by an increase in structural diversity, will lead to richer consciousness, whereas an increase in neuronal connections, if accompanied by a decrease in structural diversity, will lead to poorer consciousness. In what follows, we will discuss these studies and look into the relationship between brain structural complexity and consciousness.

\subsection{Inter-individual differences in brain structural complex- ity and consciousness}

Just as conscious experiences are unique to each individual, the brain structure of each individual is also highly unique. Utilizing the inter-individual differences in brain structure, studies have investigated the relationship between brain structure complexity and consciousness. Most of these studies use visual cortex to address this question, since visual cortical regions are the most variable regions in the human brain. Specifically, there exist over twenty visual cortical regions in the human brain, accounting for one-third of the brain volume (Silver \& Kastner, 2009; Wandell et al., 2007; Wandell \& Winawer, 2015). Although on average these regions account for one-third of the brain volume, their exact proportion in the brain and their exact volume can differ across healthy human adults over threefolds, which is far greater than the inter-individual difference in other regional volumes or the total brain volume (Andrews et al., 1997; Song, Schwarzkopf, Kanai, et al., 2011).

The volume of visual cortex is determined by two genetically independent factors, visual cortical surface area and visual cortical thickness (Chen et al., 2011; Joyner et al., 2009; Panizzon et al., 2009; Song et al., 2015). Visual cortical surface area affects the number of cortical columns per cortical region. As neurons in different cortical columns exhibit distinct response properties and distinct ontogenetic origins (Figure 6), having a larger visual cortical surface area and a larger number of cortical columns per cortical region will result in a higher level of structural diversity and functional diversity (Ko et al., 2011; Rakic, 1988; Yu et al., 2009). Visual cortical thickness, on the other hand, affects the number of neurons per cortical column. As neurons in the same cortical column exhibit similar response properties and similar ontogenetic origins (Figure 6), having a larger cortical thickness and a larger number of neurons per cortical column will instead result in a 
lower level of structural diversity and functional diversity (Ko et al., 2011; Rakic, 1988; Yu et al., 2009).

Because a higher level of structural diversity is associated with a larger visual cortical surface area but a smaller visual cortical thickness, the two hypotheses, "less is more" versus "the more the better," would make opposite predictions about the relationship between visual cortical structure and visual consciousness. Based on the hypothesis "less is more," one would predict the richness of visual consciousness to co-vary with the level of structural diversity and as such, correlate positively with visual cortical surface area but negatively with visual cortical thickness. By contrast, based on the hypothesis "the more the better," one would predict the richness of visual consciousness to co-vary with the sheer volume of visual cortex and therefore correlate positively with both visual cortical surface area and visual cortical thickness.

The empirical evidences so far have supported the hypothesis "less is more." Compared to individuals with a smaller visual cortical surface area, individuals with a larger visual cortical surface area tend to have richer visual consciousness, reflected both in the level of differentiation and in the level of integration (Figure 6). Those individuals are able to discriminate finer differences between visual inputs, which indicates more differentiated visual experiences (Song et al., 2015; Song, Schwarzkopf, \& Rees, 2013). They also report less perceptual distortion in visual contextual illusions, which indicates more integrated and structured visual experiences (Schwarzkopf et al., 2011; Song, Schwarzkopf, \& Rees, 2011; Song, Schwarzkopf, Lutti, et al., 2013; Song, Schwarzkopf, \& Rees, 2013; Song \& Rees, 2018). Notably, the exact opposite relationship was observed between visual cortical thickness and visual consciousness (Figure 6), where a larger visual cortical thickness is associated with less differentiated, less integrated visual experiences (Song et al., 2015).

The impacts of visual cortical structure on visual consciousness are recapitulated in visual neuronal functions (Figure 6). As the surface area of a visual cortical region increases, individual cortical columns in this region tend to respond to smaller, more specific ranges of visual field locations, and different cortical columns to less overlapping, more distinct ranges of visual field locations, which indicates a higher level of differentiation (Song et al., 2015); at the same time, the interactions between these cortical columns are more structured, with stronger interactions between functionally similar cortical columns, and weaker interactions between functionally distinct cortical columns, which indicates a higher level of integration. The exact opposite pattern was observed for visual cortical thickness. As the thickness of a visual cortical region increases, individual cortical columns in the region tend to respond to larger, less specific ranges of visual field locations, and different cortical columns to less distinct, more overlapping ranges of visual field locations, which indicates a lower level of differentiation (Song et al., 2015); moreover, the interactions between these cortical columns are less structured, which indicates a lower level of integration. 
Thus, an optimal cortical architecture is constituted of a larger cortical surface area (more cortical columns per cortical region) but a smaller cortical thickness (less neurons per cortical column), and it is not the sheer volume but the structural diversity that matters. By distributing neurons into different cortical columns, this cortical architecture maximizes the level of structural diversity, which in turn gives rise to higher structural complexity, better neuronal functionality, and richer conscious experiences. Indeed, individuals with such a cortical architecture tend to have richer consciousness: they can discriminate finer differences between visual inputs (higher level of differentiation) and experience less perceptual distortion in visual contextual illusions (higher level of integration); neurons in such a cortical architecture also exhibit better functionality: they can respond to less overlapping, more distinct ranges of visual field locations (higher level of differentiation) and have more structured interactions (higher level of integration).

\subsection{Intra-individual changes in brain structural complexity and consciousness}

A remarkable feature of the human brain is its adaptability and plasticity. Changes in brain structure occur not only when one is awake and interacting with the external world, but also when one is asleep (Bernardi et al., 2016; Cirelli, 2013; Song et al., 2017; Song \& Tagliazucchi, 2020; Tononi \& Cirelli, 2014; Vivo et al., 2017). Utilizing the intra-individual changes in brain structure across the sleep-wake cycle, studies have investigated the relationship between brain structure complexity and consciousness.

Specifically, during wakefulness, the brain is constantly interacting with the external world and its activity is driven primarily by inputs from the external world. The external inputs tend to co-activate different neurons, regardless of whether these neurons are previously unconnected, sparsely connected, or densely connected. The neuronal co-activation, in turn, will lead to a general increase in the number and strength of neuronal connections across the brain (Cirelli, 2013; Song \& Tagliazucchi, 2020; Tononi \& Cirelli, 2014). These wake-associated changes in brain structure are not sustainable (Figure 7): as neurons across the brain all get connected and neuronal connections across the brain all become saturated, the structural diversity between neurons and the structural diversity between neuronal connections will decrease; moreover, if the increase in neuronal connections continues without limits, for example due to prolonged wakefulness, the brain will eventually use up biological material to build, physical space to accommodate, and metabolic energy to support any further connections.

By contrast, during sleep, the brain is disconnected from the external world and its activity is driven primarily by itself. The self-driven neuronal activity exhibits spontaneous alternations between periods of intense firing and periods of silence, which in turn will lead to the pruning of weak neuronal connections and the stabilization of strong neuronal connections (Cirelli, 2013; Song \& Tagliazucchi, 2020; 


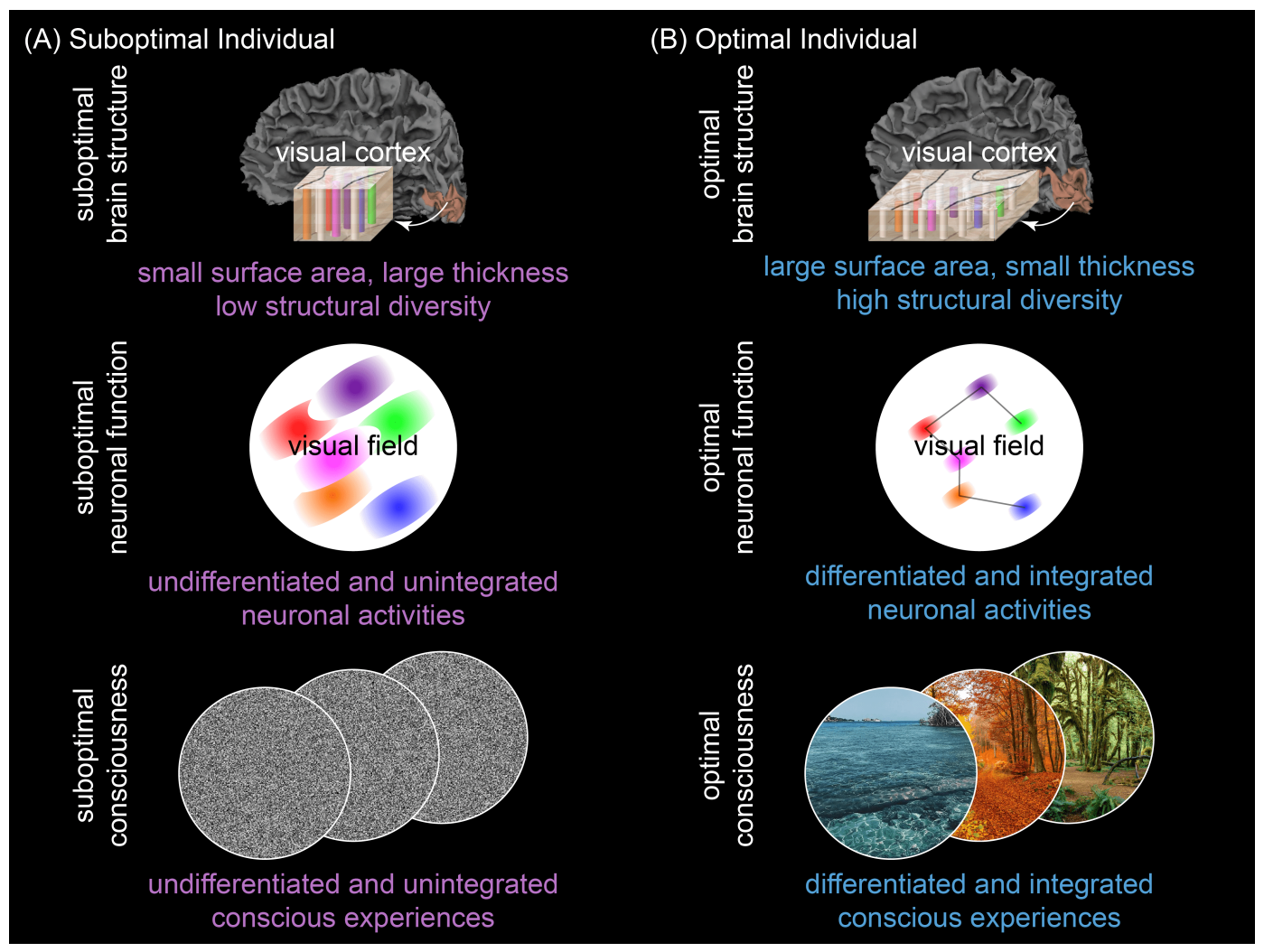

Figure 6: Inter-individual Differences in Brain Structural Complexity and Consciousness. Utilizing the inter-individual differences in cortical structure, studies have investigated the relationship between brain structure complexity and consciousness. The cortical structure is characterized by two independent factors, cortical surface area and cortical thickness. Cortical surface area affects the number of cortical columns per cortical region, and cortical thickness the number of neurons per cortical column. Since neurons in different cortical columns exhibit distinct response properties and distinct ontogenetic origins, whereas neurons in the same cortical column exhibit similar response properties and similar ontogenetic origins, having a larger cortical surface area (more cortical columns) and a smaller cortical thickness (less neurons per cortical column) can maximize the level of structural diversity in a cortical region, which can in turn give rise to higher structural complexity, better neuronal functionality, and richer conscious experiences. Indeed, individuals with such a visual cortical structure were found to have richer conscious experiences: they can discriminate finer differences between visual inputs (higher differentiation) and experience less distortion in visual illusions (higher integration); neurons in such a visual cortical structure also exhibit better functionality: they respond to less overlapping, more distinct ranges of visual field locations (higher differentiation) and have more structured interactions (higher integration).

Tononi \& Cirelli, 2014). These sleep-associated changes in brain structure are beneficial (Figure 7): as the weak connections get pruned and the strong connections get stabilized, the structural diversity between neurons and the structural diversity between neuronal connections will increase; moreover, by pruning the weak 
connections, the brain will free up biological material to build, physical space to accommodate, and metabolic energy to support new connections.

Because the number of neuronal connections and the level of structural diversity exhibit opposite changes across the sleep-wake cycle, with the former increasing during wakefulness and decreasing after sleep, whereas the latter decreasing during wakefulness and increasing after sleep, the two hypotheses, "less is more" versus "the more the better," would make opposite predictions about the impacts of these structural changes on consciousness. Based on the hypothesis "less is more," one would predict the richness of conscious experiences to co-vary with the level of structural diversity and therefore decrease over the course of wakefulness but rebound after sleep. By contrast, based on the hypothesis "the more the better," one would predict the richness of conscious experiences to co-vary with the sheer number of neuronal connections and therefore increase over the course of wakefulness but decrease after sleep.

So far, the empirical evidences have supported the hypothesis "less is more." Over the course of prolonged wakefulness, impairments in consciousness are often reported, including abnormal sensory experiences such as sensory distortion or sensory hallucination, difficulties in emotion regulation such as emotional overwhelm or emotional insensitivity, and cloudiness in thinking such as delusional thoughts or paranoid thoughts (Krause et al., 2017). These impairments are reversible by sleep, during which the wake-associated changes in brain structure are also reversed (Cirelli, 2013; Song \& Tagliazucchi, 2020; Tononi \& Cirelli, 2014). The homeostatic changes in brain structure and conscious experiences hint towards a positive correlation between the level of structural diversity and the richness of conscious experiences but a negative correlation between the sheer number of neuronal connections and the richness of conscious experiences.

Therefore, having more neuronal connections is not necessarily beneficial for the brain or for consciousness. On the contrary, an increase in neuronal connections can lead to impaired brain functionality and impaired consciousness, if the increase is accompanied by a decrease in structural diversity. Such adverse changes in brain structure in fact occur on a day-to-day basis, as the price that we pay for being awake. By pruning the excessive neuronal connections and restoring the structural diversity, sleep plays an essential role in the homeostatic optimization of brain structure and the homeostatic regulation of brain functionality and consciousness.

\section{Summary and future perspectives}

In this article, we reviewed the contributions of advanced brain imaging techniques towards uncovering brain structural complexity and its relationship to consciousness. Over the past decade, advances in magnetic resonance imaging have made it possible to measure the biophysical structure of human brain, from the morphology of neurons and neuronal connections to the gross anatomy of brain 


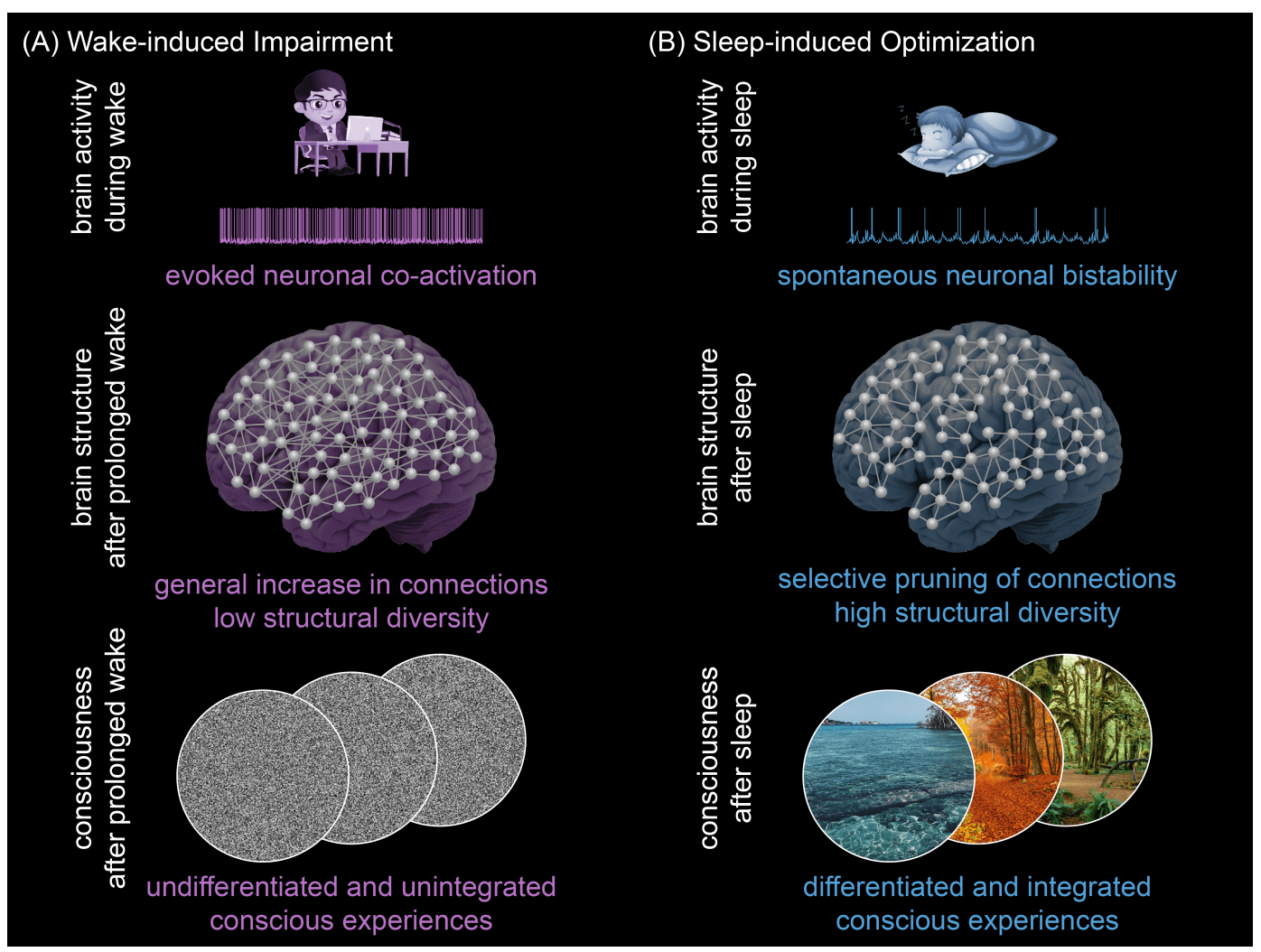

Figure 7: Intra-individual Changes in Brain Structural Complexity and Consciousness. Utilizing the intra-individual changes in brain structure across the sleep-wake cycle, studies have investigated the relationship between brain structure complexity and consciousness. (A) During wakefulness, the brain activity is driven primarily by inputs from the external world. The external inputs will co-activate different neurons, leading to a general increase in neuronal connections across the brain. As neurons across the brain all get connected and neuronal connections across the brain all become saturated, the structural diversity between neurons or neuronal connections will decrease, which will result in the impairments of brain functionality and consciousness commonly observed after prolonged wakefulness. (B) During sleep, the brain activity is driven primarily by the brain itself. The self-driven neuronal activity exhibits spontaneous alternations between periods of intense firing and periods of silence, which will lead to the pruning of weak connections and the stabilization of strong connections. As the weak connections get pruned and the strong connections get stabilized, the structural diversity between neurons or neuronal connections will increase, resulting in the restoration of brain functionality and consciousness commonly observed after sleep.

regions, in-vivo and non-invasively. Using advanced brain imaging techniques, studies are able to identify the structural features key to consciousness and explore what constitutes an optimal brain architecture for consciousness.

It was discovered that the structural diversity between neurons (cell diversity) and the topology of neuronal connections (cell-cell interaction), as opposed to the sheer number of neurons (cell number) or the sheer volume of brain (organ size), 
are the key features that distinguish the brain from other organs, underlie brain structural complexity, enhance brain functionality, and give rise to consciousness. When the structural diversity between neurons is high and the connections between neurons follow a modular topology, neurons will become functionally differentiable and at the same time, functionally integrable with each other. The high levels of differentiation and integration, in turn, enable the brain to produce a large set of differentiated yet integrated activity patterns, and subsequently, a large repertoire of differentiated yet integrated conscious experiences, from the smallest number of neurons and neuronal connections. If neurons are functionally identical to each other, for example as a result of lacking structural diversity or as a result of being over-connected, they will be fully synchronized in their activities and fail to produce differentiated activity patterns; without differentiation, consciousness will be reduced to a repertoire of identical, undifferentiable experiences. If neurons are not functionally integrable with one another, for example as a result of being under-connected, they will fail to produce structured and integrated activity patterns; without integration, consciousness will be reduced to a repertoire of unstructured, unintegrated experiences.

Therefore, an optimal brain architecture for consciousness is not necessarily constituted of a larger volume, more neurons, or more neuronal connections ("the more the better"); on the contrary, it is one where the largest repertoire of conscious experiences is generated from the smallest number of neurons and neuronal connections, at the minimal cost of biological material, physical space, and metabolic energy ("less is more"). The idea "less is more" may appear counterintuitive. However, it has received support from a number of studies, thanks to the advances in brain imaging techniques. These studies reveal that across individuals, those with a smaller brain volume but a higher structural diversity tend to have richer conscious experiences than those with a larger brain volume but a lower structural diversity; moreover, within individuals, a reduction in neuronal connections, if accompanied by an increase in structural diversity, will lead to richer conscious experiences, whereas an increase in neuronal connections, if accompanied by a decrease in structural diversity, will lead to poorer conscious experiences.

Despite all these progress made towards understanding the structural basis of consciousness, many open questions remain. For example, how the structural diversity between neurons is generated at the first place remains largely unclear. According to Darwinism, diversity is a basic property of biological systems. It is generated at the genotype level by random genetic accident, amplified at the phenotype level by gene-environment interaction, and reinforced at the evolution level by natural selection. Darwin proposed that the diversity between individuals can enhance the adaptivity and the resilience of the population (Forsman, 2014; Norberg et al., 2001), as the diversity enables different individuals of the population to perform mutually incompatible functions (akin to differentiation) in a collaborative way (akin to integration). Given its functional benefits, the trait 
of inter-individual diversity is favoured, preserved and reinforced by evolution, which underlies the biodiversity in our current world.

Possibly, just as the diversity within a population between its individuals can enhance the adaptivity of the population, the diversity within an individual between its cells can enhance the adaptivity of the individual and is therefore similarly reinforced by evolution (Bryant \& Mostov, 2008). This explains the increase in cellular diversity along evolution, from unicellular organism where a single cell carries out all functions, to multicellular organism where different cells carry out distinct functions in a collaborative way (Bryant \& Mostov, 2008). This also explains why evolutionarily younger organs tend to have higher cellular diversity than evolutionarily older organs, and why the brain has the highest cellular diversity among all organs (Bail et al., 2021; Bakken et al., 2020; Bryant \& Mostov, 2008). The fact that the brain surpasses other organs not in absolute size but in cellular diversity provides further evidence for the idea "less is more" and against the conventional wisdom "the more the better."

Moving forward, future research may apply single cell sequencing to investigate the origin of structural diversity between neurons. This technique enables simultaneous profiling of genome, epigenome, transcriptome, and proteome at the resolution of single cells (Eberwine et al., 2014; Macaulay et al., 2017). Using it, studies have unveiled an incredible amount of genomic diversity between neurons (Darmanis et al., 2015; Lake et al., 2016), which overturned the conventional view that different neurons in the same brain all carry the same genome, and their structural diversity arises epigenomically, transcriptomically, or proteomically from how that genome is expressed. The technique of single cell sequencing may be applied to map the neuronal diversity in different species (Bail et al., 2021; Bakken et al., 2020) and investigate how that relates with the emergence of consciousness.

\section{Acknowledgments}

This work was supported by Wellcome Trust Grant 209192/Z/17/Z (CS) and H2020 MSCA COFUND Grant 663830-CU119 (CS).

\section{References}

Amunts, K., Lenzen, M., Friederici, A. D., Schleicher, A., Morosan, P., Palomero-Gallagher, N., \& Zilles, K. (2010). Broca's region: Novel organizational principles and multiple receptor mapping. PLoS Biology, 8(9), e1000489. https://doi.org/ 10.1371/journal.pbio.1000489

Amunts, K., \& Zilles, K. (2015). Architectonic mapping of the human brain beyond brodmann. Neuron, 88(6), $1086-1107$. https://doi.org/10.1016/j.neuron.2015.12.001

Andrews, T. J., Halpern, S. D., \& Purves, D. (1997). Correlated size variations in human visual cortex, lateral geniculate nucleus, and optic tract. The fournal of Neuroscience, 17(8), 2859-2868. https://doi.org/10.1523/JNEUROSCI.17-0802859.1997

Arancibia, I. L., Ford, M. C., Cossell, L., Ishida, K., Tohyama, K., \& Attwell, D. (2017). Node of ranvier length as a potential regulator of myelinated axon conduction speed. eLife, 6. https://doi.org/10.7554/eLife.23329

Assaf, Y., \& Basser, P. J. (2005). Composite hindered and restricted model of diffusion (CHARMED) MR imaging of the human brain. NeuroImage, 27(1), 48-58. https://doi.org/10.1016/j.neuroimage.2005.03.042 
Assaf, Y., Blumenfeld, T., Yovel, Y., \& Basser, P. J. (2008). Axcaliber a method for measuring axon diameter distribution from diffusion MRI. Magnetic Resonance in Medicine, 59(6), 1347-1354. https://doi.org/10.1002/mrm.21577

Bail, R. L., Bonafina, A., Espuny-Camacho, I., \& Nguyen, L. (2021). Learning about cell lineage, cellular diversity and evolution of the human brain through stem cell models. Current Opinion in Neurobiology, 66, 166-177. https://doi.org/https: //doi.org/10.1016/j.conb.2020.10.018

Bakken, T. E., Jorstad, N. L., Hu, Q., Lake, B. B., Tian, W., Kalmbach, B. E., Crow, M., Hodge, R. D., Krienen, F. M., Sorensen, S. A., Eggermont, J., Yao, Z., Aevermann, B. D., Aldridge, A. I., Bartlett, A., Bertagnolli, D., Casper, T., Castanon, R. G., Crichton, K., ... Lein, E. S. (2020). Evolution of cellular diversity in primary motor cortex of human, marmoset monkey, and mouse. bioRxiv, 2020.03.31.016972. https://doi.org/10.1101/2020.03.31.016972

Bassett, D. S., \& Gazzaniga, M. S. (2011). Understanding complexity in the human brain. Trends in Cognitive Sciences, 15(5), 200-209. https://doi.org/10.1016/j.tics.2011.03.006

Bekkers, J. M. (2011). Pyramidal neurons. Current Biology, 21(24), R975. https://doi.org/10.1016/j.cub.2011.10.037

Bernardi, G., Cecchetti, L., Siclari, F., Buchmann, A., Yu, X., Handjaras, G., Bellesi, M., Ricciardi, E., Kecskemeti, S. R., Riedner, B. A., Alexander, A. L., Benca, R. M., Ghilardi, M. F., Pietrini, P., Cirelli, C., \& Tononi, G. (2016). Sleep reverts changes in human gray and white matter caused by wake-dependent training. NeuroImage, 129, 367-377. https://doi.org/10.1016/j.neuroimage.2016.01.020

Boly, M., Massimini, M., Tsuchiya, N., Postle, B. R., Koch, C., \& Tononi, G. (2017). Are the neural correlates of consciousness in the front or in the back of the cerebral cortex? Clinical and neuroimaging evidence. The fournal of Neuroscience: The Official fournal of the Society for Neuroscience, 37(40), 9603-9613. https://doi.org/10.1523/JNEUROSCI.3218-16.2017

Brown, K. M., Gillette, T. A., \& Ascoli, G. A. (2008). Quantifying neuronal size: Summing up trees and splitting the branch difference. Seminars in Cell \& Developmental Biology, 19(6), 485-493. https://doi.org/10.1016/j.semcdb.2008.08.005

Bryant, D. M., \& Mostov, K. E. (2008). From cells to organs: Building polarized tissue. Nature Reviews Molecular Cell Biology, 9(11), 887-901. https://doi.org/10.1038/nrm2523

Bullmore, E., \& Sporns, O. (2009). Complex brain networks: Graph theoretical analysis of structural and functional systems. Nature Reviews Neuroscience, 10(3), 186-198. https://doi.org/10.1038/nrn2575

Chen, C.-H., Panizzon, M. S., Eyler, L. T., Jernigan, T. L., Thompson, W., Fennema-Notestine, C., Jak, A. J., Neale, M. C., Franz, C. E., Hamza, S., Lyons, M. J., Grant, M. D., Fischl, B., Seidman, L. J., Tsuang, M. T., Kremen, W. S., \& Dale, A. M. (2011). Genetic influences on cortical regionalization in the human brain. Neuron, 72(4), 537-544. https: //doi.org/10.1016/j.neuron.2011.08.021

Chereau, R., Saraceno, G. E., Angibaud, J., Cattaert, D., \& Nägerl, U. V. (2017). Superresolution imaging reveals activitydependent plasticity of axon morphology linked to changes in action potential conduction velocity. Proceedings of the National Academy of Sciences, 114(6), 1401-1406. https://doi.org/10.1073/pnas.1607541114

Chklovskii, D. (2004). Synaptic connectivity and neuronal morphology: Two sides of the same coin. Neuron, 43(5), 609-617. https://doi.org/10.1016/S0896-6273(04)00498-2

Cirelli, C. (2013). Sleep and synaptic changes. Current Opinion in Neurobiology, 23(5), 841-846. https://doi.org/10.1016/j.co nb.2013.04.001

Clarke, S., \& Miklossy, J. (1990). Occipital cortex in man: Organization of callosal connections, related myelo- and cytoarchitecture, and putative boundaries of functional visual areas. The fournal of Comparative Neurology, 298(2), 188-214. https://doi.org/10.1002/cne.902980205

Clune, J., Mouret, J.-B., \& Lipson, H. (2013). The evolutionary origins of modularity. Proceedings of the Royal Society B: Biological Sciences, 280(1755), 20122863. https://doi.org/10.1098/rspb.2012.2863

Crick, F., \& Koch, C. (2003). A framework for consciousness. Nature Neuroscience, 6(2), 119-126. https://doi.org/10.1038/nn 0203-119

Dale, A. M., Fischl, B., \& Sereno, M. I. (1999). Cortical surface-based analysis I: Segmentation and surface reconstruction. NeuroImage, 9, 179-194. https://doi.org/10.1006/nimg.1998.0395

Darmanis, S., Sloan, S. A., Zhang, Y., Enge, M., Caneda, C., Shuer, L. M., Hayden Gephart, M. G., Barres, B. A., \& Quake, S. R. (2015). A survey of human brain transcriptome diversity at the single cell level. Proceedings of the National Academy of Sciences, 112(23), 7285-7290. https://doi.org/10.1073/pnas.1507125112

Eberwine, J., Sul, J.-Y., Bartfai, T., \& Kim, J. (2014). The promise of single-cell sequencing. Nature Methods, 11(1), 25-27. https://doi.org/10.1038/nmeth.2769

Edwards, L. J., Kirilina, E., Mohammadi, S., \& Weiskopf, N. (2018). Microstructural imaging of human neocortex in vivo. NeuroImage, 182, 184-206. https://doi.org/10.1016/j.neuroimage.2018.02.055

Fink, S. B. (2016). A deeper look at the "neural correlate of consciousness." Frontiers in Psychology, 7, 1044. https://doi.org/ 10.3389/fpsyg.2016.01044

Song, C. (2021). Brain structural complexity and consciousness. Philosophy and the Mind Sciences,

2, 6. https://doi.org/10.33735/phimisci.2021.9185

(G) (1) The author(s). https://philosophymindscience.org ISSN: 2699-0369 
Firmin, L., Field, P., Maier, M. A., Kraskov, A., Kirkwood, P. A., Nakajima, K., Lemon, R. N., \& Glickstein, M. (2014). Axon diameters and conduction velocities in the macaque pyramidal tract. fournal of Neurophysiology, 112(6), 1229-1240. https://doi.org/10.1152/jn.00720.2013

Fischl, B., Sereno, M. I., \& Dale, A. M. (1999). Cortical surface-based analysis II: Inflation, flattening, and a surface-based coordinate system. NeuroImage, 9, 195-207. https://doi.org/10.1006/nimg.1998.0396

Forsman, A. (2014). Effects of genotypic and phenotypic variation on establishment are important for conservation, invasion, and infection biology. Proceedings of the National Academy of Sciences, 111(1), 302-307. https://doi.org/10.1073/ pnas. 1317745111

Gallos, L. K., Makse, H. A., \& Sigman, M. (2012). A small world of weak ties provides optimal global integration of selfsimilar modules in functional brain networks. Proceedings of the National Academy of Sciences, 109(8), 2825-2830. https://doi.org/10.1073/pnas.1106612109

Gilbert, C., \& Wiesel, T. (1989). Columnar specificity of intrinsic horizontal and corticocortical connections in cat visual cortex. The fournal of Neuroscience, 9(7), 2432-2442. https://doi.org/10.1523/JNEUROSCI.09-07-02432.1989

Girvan, M., \& Newman, M. E. J. (2002). Community structure in social and biological networks. Proceedings of the National Academy of Sciences, 99(12), 7821-7826. https://doi.org/10.1073/pnas.122653799

Glasser, M. F., Coalson, T. S., Robinson, E. C., Hacker, C. D., Harwell, J., Yacoub, E., Ugurbil, K., Andersson, J., Beckmann, C. F., Jenkinson, M., Smith, S. M., \& Van Essen, D. C. (2016). A multi-modal parcellation of human cerebral cortex. Nature, 536(7615), 171-178. https://doi.org/10.1038/nature18933

Glasser, M. F., Smith, S. M., Marcus, D. S., Andersson, J. L. R., Auerbach, E. J., Behrens, T. E. J., Coalson, T. S., Harms, M. P., Jenkinson, M., Moeller, S., Robinson, E. C., Sotiropoulos, S. N., Xu, J., Yacoub, E., Ugurbil, K., \& Van Essen, D. C. (2016). The human connectome project's neuroimaging approach. Nature Neuroscience, 19(9), 1175-1187. https: //doi.org/10.1038/nn.4361

Harris, J. A., Mihalas, S., Hirokawa, K. E., Whitesell, J. D., Choi, H., Bernard, A., Bohn, P., Caldejon, S., Casal, L., Cho, A., Feiner, A., Feng, D., Gaudreault, N., Gerfen, C. R., Graddis, N., Groblewski, P. A., Henry, A. M., Ho, A., Howard, R., ... Zeng, H. (2019). Hierarchical organization of cortical and thalamic connectivity. Nature, 575(7781), 195-202. https://doi.org/10.1038/s41586-019-1716-z

Hartwell, L. H., Hopfield, J. J., Leibler, S., \& Murray, A. W. (1999). From molecular to modular cell biology. Nature, 402(S6761), C47-C52. https://doi.org/10.1038/35011540

Horowitz, A., Barazany, D., Tavor, I., Bernstein, M., Yovel, G., \& Assaf, Y. (2015). In vivo correlation between axon diameter and conduction velocity in the human brain. Brain Structure and Function, 220(3), 1777-1788. https://doi.org/10.1007/ s00429-014-0871-0

Hwang, K., Bertolero, M. A., Liu, W. B., \& D'Esposito, M. (2017). The human thalamus is an integrative hub for functional brain networks. The fournal of Neuroscience, 37(23), 5594-5607. https://doi.org/10.1523/JNEUROSCI.0067-17.2017

Jones, D. K., Alexander, D. C., Bowtell, R., Cercignani, M., Dell'Acqua, F., McHugh, D. J., Miller, K. L., Palombo, M., Parker, G. J. M., Rudrapatna, U. S., \& Tax, C. M. W. (2018). Microstructural imaging of the human brain with a super-scanner: 10 key advantages of ultra-strong gradients for diffusion MRI. NeuroImage, 182, 8-38. https://doi.org/10.1016/j.neuroi mage.2018.05.047

Joyner, A. H., J., C. R., Bloss, C. S., Bakken, T. E., Rimol, L. M., Melle, I., Agartz, I., Djurovic, S., Topol, E. J., Schork, N. J., Andreassen, O. A., \& Dale, A. M. (2009). A common MECP2 haplotype associates with reduced cortical surface area in humans in two independent populations. Proceedings of the National Academy of Sciences, 106(36), 15483-15488. https://doi.org/10.1073/pnas.0901866106

Kaas, J. H. (2012). Evolution of columns, modules, and domains in the neocortex of primates. Proceedings of the National Academy of Sciences, 109(Supplement_1), 10655-10660. https://doi.org/10.1073/pnas.1201892109

Kaas, J. H. (2000). Why is brain size so important: Design problems and solutions as neocortex gets bigger or smaller. Brain and Mind, 7-23. https://doi.org/10.1023/A:1010028405318

Kanai, R., \& Rees, G. (2011). The structural basis of inter-individual differences in human behaviour and cognition. Nature Reviews. Neuroscience, 12(4), 231-242. https://doi.org/10.1038/nrn3000

Kashtan, N., \& Alon, U. (2005). Spontaneous evolution of modularity and network motifs. Proceedings of the National Academy of Sciences, 102(39), 13773-13778. https://doi.org/10.1073/pnas.0503610102

Ko, H., Hofer, S. B., Pichler, B., Buchanan, K. A., Sjöström, P. J., \& Mrsic-Flogel, T. D. (2011). Functional specificity of local synaptic connections in neocortical networks. Nature, 473(7345), 87-91. https://doi.org/10.1038/nature09880

Koch, C. (2019). The feeling of life itself: Why consciousness is widespread but can't be computed. The MIT Press.

Koch, C., Massimini, M., Boly, M., \& Tononi, G. (2016). Neural correlates of consciousness: Progress and problems. Nature Reviews Neuroscience, 17(5), 307-321. https://doi.org/10.1038/nrn.2016.22

Krause, A. J., Simon, E. B., Mander, B. A., Greer, S. M., Saletin, J. M., Goldstein-Piekarski, A. N., \& Walker, M. P. (2017). The sleep-deprived human brain. Nature Reviews Neuroscience, 18(7), 404-418. https://doi.org/10.1038/nrn.2017.55

Song, C. (2021). Brain structural complexity and consciousness. Philosophy and the Mind Sciences, 2, 6. https://doi.org/10.33735/phimisci.2021.9185

(G) (1) The author(s). https://philosophymindscience.org ISSN: 2699-0369 
Lake, B. B., Ai, R., Kaeser, G. E., Salathia, N. S., Yung, Y. C., Liu, R., Wildberg, A., Gao, D., Fung, H.-L., Chen, S., Vijayaraghavan, R., Wong, J., Chen, A., Sheng, X., Kaper, F., Shen, R., Ronaghi, M., Fan, J.-B., Wang, W., ... Zhang, K. (2016). Neuronal subtypes and diversity revealed by single-nucleus RNA sequencing of the human brain. Science, 352(6293), 1586-1590. https://doi.org/10.1126/science.aaf1204

Lau, H., \& Rosenthal, D. (2011). Empirical support for higher-order theories of conscious awareness. Trends in Cognitive Sciences, 15(8), 365-373. https://doi.org/10.1016/j.tics.2011.05.009

Le Bihan, D., \& Iima, M. (2015). Diffusion MRI: What water tells us about biological tissues. PLOS Biology, 13(7), e1002203. https://doi.org/10.1371/journal.pbio.1002203

Lisman, J. E. (2017). Locke's view of the hard problem of consciousness and its implications for neuroscience and computer science. Frontiers in Psychology, 8, 1069. https://doi.org/10.3389/fpsyg.2017.01069

Macaulay, I. C., Ponting, C. P., \& Voet, T. (2017). Single-cell multiomics: Multiple measurements from single cells. Trends in Genetics, 33(2), 155-168. https://doi.org/10.1016/j.tig.2016.12.003

Mountcastle, V. (1997). The columnar organization of the neocortex. Brain, 120(4), 701-722. https://doi.org/10.1093/brain/ 120.4.701

Newman, M. E. J. (2006). Modularity and community structure in networks. Proceedings of the National Academy of Sciences, 103(23), 8577-8582. https://doi.org/10.1073/pnas.0601602103

Norberg, J., Swaney, D. P., Dushoff, J., Lin, J., Casagrandi, R., \& Levin, S. A. (2001). Phenotypic diversity and ecosystem functioning in changing environments: A theoretical framework. Proceedings of the National Academy of Sciences, 98(20), 11376-11381. https://doi.org/10.1073/pnas.171315998

Odegaard, B., Knight, R. T., \& Lau, H. (2017). Should a few null findings falsify prefrontal theories of conscious perception? The Fournal of Neuroscience: The Official Fournal of the Society for Neuroscience, 37(40), 9593-9602. https://doi.org/10 .1523/JNEUROSCI.3217-16.2017

Oizumi, M., Albantakis, L., \& Tononi, G. (2014). From the phenomenology to the mechanisms of consciousness: Integrated information theory 3.0. PLoS Computational Biology, 10(5), e1003588. https://doi.org/10.1371/journal.pcbi.1003588

Palombo, M., Ligneul, C., Najac, C., Le Douce, J., Flament, J., Escartin, C., Hantraye, P., Brouillet, E., Bonvento, G., \& Valette, J. (2016). New paradigm to assess brain cell morphology by diffusion-weighted MR spectroscopy in vivo. Proceedings of the National Academy of Sciences, 113(24), 6671-6676. https://doi.org/10.1073/pnas.1504327113

Palombo, M., Shemesh, N., Ronen, I., \& Valette, J. (2018). Insights into brain microstructure from in vivo DW-MRS. NeuroImage, 182, 97-116. https://doi.org/10.1016/j.neuroimage.2017.11.028

Palomero, N., \& Zilles, K. (2019). Cortical layers: Cyto-, myelo-, receptor- and synaptic architecture in human cortical areas. NeuroImage, 197, 716-741. https://doi.org/10.1016/j.neuroimage.2017.08.035

Pan, R. K., \& Sinha, S. (2009). Modularity produces small-world networks with dynamical time-scale separation. Europhysics Letters, 85(6), 68006. https://doi.org/10.1209/0295-5075/85/68006

Panda, A., Mehta, B. B., Coppo, S., Jiang, Y., Ma, D., Seiberlich, N., Griswold, M. A., \& Gulani, V. (2017). Magnetic resonance fingerprinting: An overview. Current Opinion in Biomedical Engineering, 3, 56-66. https://doi.org/10.1016/j.cobme.20 17.11.001

Panizzon, M. S., Fennema-Notestine, C., Eyler, L. T., Jernigan, T. L., Prom-Wormley, E., Neale, M., Jacobson, K., Lyons, M. J., Grant, M. D., Franz, C. E., Xian, H., Tsuang, M., Fischl, B., Seidman, L., Dale, A., \& Kremen, W. S. (2009). Distinct genetic influences on cortical surface area and cortical thickness. Cerebral Cortex, 19(11), 2728-2735. https: //doi.org/10.1093/cercor/bhp026

Pearce, J. M. S. (2013). The neuroanatomy of herophilus. European Neurology, 69(5), 292-295. https://doi.org/10.1159/0003 46232

Rakic, P. (1988). Specification of cerebral cortical areas. Science (New York, N.Y.), 241(4862), 170-176. https://doi.org/10.112 6/science. 3291116

Ravasz, E. (2002). Hierarchical organization of modularity in metabolic networks. Science, 297(5586), 1551-1555. https: //doi.org/10.1126/science.1073374

Schwarzkopf, D. S., Song, C., \& Rees, G. (2011). The surface area of human V1 predicts the subjective experience of object size. Nature Neuroscience, 14(1), 28-30. https://doi.org/10.1038/nn.2706

Shipp, S. (2005). The importance of being agranular: A comparative account of visual and motor cortex. Philosophical Transactions of the Royal Society B: Biological Sciences, 360(1456), 797-814. https://doi.org/10.1098/rstb.2005.1630

Siclari, F., LaRocque, J., Postle, B., \& Tononi, G. (2013). Assessing sleep consciousness within subjects using a serial awakening paradigm. Frontiers in Psychology, 4, 542. https://doi.org/10.3389/fpsyg.2013.00542

Silver, M. A., \& Kastner, S. (2009). Topographic maps in human frontal and parietal cortex. Trends in Cognitive Sciences, 13(11), 488-495. https://doi.org/10.1016/j.tics.2009.08.005

Sole, R., \& Valverde, S. (2006). Are network motifs the spandrels of cellular complexity? Trends in Ecology \& Evolution, 21(8), 419-422. https://doi.org/10.1016/j.tree.2006.05.013 
Song, C., Haun, A. M., \& Tononi, G. (2017). Plasticity in the structure of visual space. eNeuro, 4(3). https://doi.org/10.1523/ ENEURO.0080-17.2017

Song, C., Havlin, S., \& Makse, H. A. (2005). Self-similarity of complex networks. Nature, 433(7024), 392-395. https://doi.or $\mathrm{g} / 10.1038 /$ nature 03248

Song, C., \& Rees, G. (2018). Intra-hemispheric integration underlies perception of tilt illusion. NeuroImage, 175, 80-90. https://doi.org/10.1016/j.neuroimage.2018.03.073

Song, C., Schwarzkopf, D. S., Kanai, R., \& Rees, G. (2015). Neural population tuning links visual cortical anatomy to human visual perception. Neuron, 85(3), 641-656. https://doi.org/10.1016/j.neuron.2014.12.041

Song, C., Schwarzkopf, D. S., Kanai, R., \& Rees, G. (2011). Reciprocal anatomical relationship between primary sensory and prefrontal cortices in the human brain. The fournal of Neuroscience: The Official fournal of the Society for Neuroscience, 31(26), 9472-9480. https://doi.org/10.1523/JNEUROSCI.0308-11.2011

Song, C., Schwarzkopf, D. S., Lutti, A., Li, B., Kanai, R., \& Rees, G. (2013). Effective connectivity within human primary visual cortex predicts interindividual diversity in illusory perception. The fournal of Neuroscience: The Official fournal of the Society for Neuroscience, 33(48), 18781-18791. https://doi.org/10.1523/JNEUROSCI.4201-12.2013

Song, C., Schwarzkopf, D. S., \& Rees, G. (2011). Interocular induction of illusory size perception. BMC Neuroscience, 12, 27. https://doi.org/10.1186/1471-2202-12-27

Song, C., Schwarzkopf, D. S., \& Rees, G. (2013). Variability in visual cortex size reflects tradeoff between local orientation sensitivity and global orientation modulation. Nature Communications, 4, 2201. https://doi.org/10.1038/ncomms3201

Song, C., \& Tagliazucchi, E. (2020). Linking the nature and functions of sleep: Insights from multimodal imaging of the sleeping brain. Current Opinion in Physiology, 15, 29-36. https://doi.org/10.1016/j.cophys.2019.11.012

Sporns, O. (2013). Network attributes for segregation and integration in the human brain. Current Opinion in Neurobiology, 23(2), 162-171. https://doi.org/10.1016/j.conb.2012.11.015

Sporns, O., \& Betzel, R. F. (2016). Modular brain networks. Annual Review of Psychology, 67(1), 613-640. https://doi.org/10 .1146/annurev-psych-122414-033634

Sporns, O., Tononi, G., \& Kötter, R. (2005). The human connectome: A structural description of the human brain. PLoS Computational Biology, 1(4), e42. https://doi.org/10.1371/journal.pcbi.0010042

Sutherland, S. (1995). The Macmillan dictionary of psychology. Macmillan International Higher Education.

Tononi, G. (1998). Complexity and coherency: Integrating information in the brain. Trends in Cognitive Sciences, 2(12), 474-484. https://doi.org/10.1016/S1364-6613(98)01259-5

Tononi, G., Boly, M., Massimini, M., \& Koch, C. (2016). Integrated information theory: From consciousness to its physical substrate. Nature Reviews Neuroscience, 17(7), 450-461. https://doi.org/10.1038/nrn.2016.44

Tononi, G., \& Cirelli, C. (2014). Sleep and the price of plasticity: From synaptic and cellular homeostasis to memory consolidation and integration. Neuron, 81(1), 12-34. https://doi.org/10.1016/j.neuron.2013.12.025

Tononi, G., \& Edelman, G. M. (1998). Consciousness and complexity. Science, 282, 1846-1851. https://doi.org/10.1126/scie nce.282.5395.1846

Tononi, G., Sporns, O., \& Edelman, G. M. (1999). Measures of degeneracy and redundancy in biological networks. Proceedings of the National Academy of Sciences, 96(6), 3257-3262. https://doi.org/10.1073/pnas.96.6.3257

Tononi, G., Sporns, O., \& Edelman, G. M. (1994). A measure for brain complexity: Relating functional segregation and integration in the nervous system. Proceedings of the National Academy of Sciences, 91(11), 5033-5037. https://doi.or $\mathrm{g} / 10.1073 /$ pnas.91.11.5033

Tubbs, R. S. (2015). Anatomy is to physiology as geography is to history. Clinical Anatomy, 28(2), 151. https://doi.org/10.1 $002 /$ ca.22526

Vivo, L. de, Bellesi, M., Marshall, W., Bushong, E. A., Ellisman, M. H., Tononi, G., \& Cirelli, C. (2017). Ultrastructural evidence for synaptic scaling across the wake/sleep cycle. Science, 355(6324), 507-510. https://doi.org/10.1126/science.aah5982

Wandell, B. A., Dumoulin, S. O., \& Brewer, A. A. (2007). Visual field maps in human cortex. Neuron, 56(2), 366-383. https://doi.org/10.1016/j.neuron.2007.10.012

Wandell, B. A., \& Winawer, J. (2015). Computational neuroimaging and population receptive fields. Trends in Cognitive Sciences, 19(6), 349-357. https://doi.org/10.1016/j.tics.2015.03.009

Weiskopf, N., Suckling, J., Williams, G., Correia, M. M., Inkster, B., Tait, R., Ooi, C., Bullmore, E. T., \& Lutti, A. (2013). Quantitative multi-parameter mapping of R1, $\mathrm{PD}\left({ }^{*}\right), \mathrm{MT}$, and $\mathrm{R} 2\left(^{*}\right)$ at $3 \mathrm{~T}$ : A multi-center validation. Frontiers in Neuroscience, 7, 95. https://doi.org/10.3389/fnins.2013.00095

Weliky, M., Kandler, K., Fitzpatrick, D., \& Katz, L. C. (1995). Patterns of excitation and inhibition evoked by horizontal connections in visual cortex share a common relationship to orientation columns. Neuron, 15(3), 541-552. https: //doi.org/10.1016/0896-6273(95)90143-4

Song, C. (2021). Brain structural complexity and consciousness. Philosophy and the Mind Sciences, 2, 6. https://doi.org/10.33735/phimisci.2021.9185

(c) (1) (c) The author(s). https://philosophymindscience.org ISSN: 2699-0369 
Windt, J. M. (2015). Dreaming a conceptual framework for philosophy of mind and empirical research. The MIT Press. http: //www.jstor.org/stable/j.ctt17kk7qt

Yu, Y.-C., Bultje, R. S., Wang, X., \& Shi, S.-H. (2009). Specific synapses develop preferentially among sister excitatory neurons in the neocortex. Nature, 458(7237), 501-504. https://doi.org/10.1038/nature07722

\section{Open Access}

This article is distributed under the terms of the Creative Commons Attribution 4.0 International License (https://creativecommons.org/licenses/by/4.0/), which permits unrestricted use, distribution, and reproduction in any medium, as long as you give appropriate credit to the original author(s) and the source, provide a link to the Creative Commons license, and indicate if changes were made. 